\title{
The influence of nitridation time on the structural properties of GaN grown on $\mathrm{Si}$ (111) substrate
}

\author{
Engin Arslan • Mustafa K. Ozturk • Özgür Duygulu • \\ Ali Arslan Kaya · Suleyman Ozcelik · Ekmel Ozbay
}

Received: 31 July 2008 / Accepted: 13 October 2008 / Published online: 30 October 2008

(C) Springer-Verlag 2008

\begin{abstract}
In the present paper, the effect of in-situ substrate nitridation time on crystalline quality of $\mathrm{GaN}$ films grown on Si (111) substrates by metal organic chemical vapor deposition (MOCVD) were investigated. A thin buffer layer of silicon nitride $\left(\mathrm{SiN}_{x}\right)$ with various thicknesses was achieved through the nitridation of substrate at different nitiridation times ranging from 0 to $660 \mathrm{~s}$. The structural characteristics, such as dislocation densities, correlation lengths of columnar crystallites, the tilt and twist of the mosaic structure, and the angles of rotational disorder, were all studied in detail by using a planar and cross-sectional view of high resolution transmission electron microscopy (HRTEM) and X-ray diffraction (HRXRD) performed at different scattering geometries. It was found that the dislocation densities, lateral coherence lengths, vertical coherence lengths, and the tilt and
\end{abstract}

E. Arslan $(\varangle) \cdot$ E. Ozbay

Nanotechnology Research Center, Department of Physics,

Department of Electrical and Electronics Engineering, Bilkent

University, Bilkent, 06800 Ankara, Turkey

e-mail: engina@bilkent.edu.tr

Fax: +90-312-2901015

M.K. Ozturk · S. Ozcelik

Department of Physics, Faculty of Science and Arts, Gazi

University, Teknikokullar, 06500 Ankara, Turkey

M.K. Ozturk

Department of Mineral Analysis and Technology, MTA, 06520

Ankara, Turkey

Ö. Duygulu

TUBITAK Marmara Research Center, Materials Institute,

P.O. Box 21 Gebze Kocaeli, 41470, Kocaeli, Turkey

A.A. Kaya

Engineering Faculty, Metallurgy and Materials Eng. Dept., Mugla

University, 48170 Mugla, Turkey twist of mosaic blocks in GaN films monotonically varies with the nitridation time. The experimental findings showed that the nitridation times had more influence on edge dislocation densities than the screw type.

PACS 61.05.Cp · 61.72.Uj · 64.70.Kg · 68.37.Lp ·

68.37.Og $\cdot 72.80 . \mathrm{Ey} \cdot 78.55 . \mathrm{Cr}$

\section{Introduction}

The large direct band-gap and good thermal stability properties of the $\mathrm{GaN}$ and related materials has enabled their potential use in applications for laser diodes (LDs), visible-blind ultraviolet (UV) detectors, and short-wave light-emitting diodes (LEDs) [1-4]. However, there is still a lack of a suitable substrate for GaN-based devices to fully exploit their superior properties. Thus far, there have been a large number of studies concerned with the growth of GaN films on highly lattice-mismatched substrates, such as sapphire [5], $\mathrm{SiC}$ [6], or Si [7]. Among these materials, silicon has many advantages compared to $\mathrm{SiC}$ and sapphire due to its high crystal quality, low cost, good electrical and thermal conductivity, and large-area size as a substrate for the growth of $\mathrm{GaN}, \mathrm{AlGaN}$ epitaxial layers [8, 9].

However, the growth of GaN films on a Si wafer is usually associated with such problems as cracking in the epitaxial layer because of large mismatches in the lattice parameters $(-16.9 \%)$ and thermal expansion coefficients (approx. 113\%) [5, 7, 10-13]. Due to a large lattice mismatch, a high density of threading dislocations on the order of $\left(10^{9}-10^{10} \mathrm{~cm}^{-2}\right)$ exists in the GaN film on silicon substrates, which significantly affects the performance of the 
GaN based devices [8]. In order to obtain crack-free highquality GaN film on Si substrate, various types of buffer layers and growth conditions as well as post-growth heat treatment processes have been proposed by different research groups [10-17]. However, the appearance of the cracks is quite random on the film, which produces significant difficulty in device applications. Therefore, the control of crack distribution for a large area film is the main issue of the present study [7-11].

It has been recently demonstrated that defect-free $\mathrm{GaN}$ on $\mathrm{Si}$ could be realized when a $\mathrm{Si}_{x} \mathrm{~N}_{y}$ buffer was used in hot wall chemical vapor deposition [14] with MOCVD [15] growth techniques. These buffer layers were achieved by nitridating the $\mathrm{Si}$ substrate with $\mathrm{N}_{2}$ flow at $900^{\circ} \mathrm{C}$ by Huang et al. [14] and at $1120^{\circ} \mathrm{C}$ by Wu-Yih Uena et al. [15]. With a similar approach, a double-buffer structure of $\mathrm{AlN} / \mathrm{Si}_{x} \mathrm{~N}_{y}$ was used to obtain high quality GaN film on Si substrate by using molecular beam epitaxy (MBE) [16]. The singlecrystalline $\mathrm{SiN}_{x}$ was obtained by introducing the active nitrogen plasma to the $\mathrm{Si}$ (111) surface at $900^{\circ} \mathrm{C}$ for approximately $30 \mathrm{~s}$. Hageman et al. [17] reported significant improvements on the optical and structural properties of the $1 \mu \mathrm{m} \mathrm{GaN}$ layer with the creation of a $\mathrm{SiN}_{x}$ intermediate layer.

Large lattice mismatch with respect to the substrate commonly causes high dislocation densities in heteroepitaxial thin films [18-21]. These types of structures are often described by the model of mosaic crystals [20, 22]. The lateral and vertical dimensions of the crystallites are given by the lateral and vertical coherence length. The angular mismatch of the crystallites causes a tilt and twist, describing the angular distribution of the crystallographic orientation of the mosaic blocks perpendicular to, and within, the growth plane. Knowledge of all these structural parameters in detail is crucial in order to understand the microstructural properties of the grown epilayers, to optimize the growth conditions, and the buffer design for better GaN film quality on Si substrate.

We investigated in detail the effect of in-situ substrate nitridation time (assumed to cause an $\mathrm{SiN}_{x}$ amorphous interlayer) on the characteristics of structural features (correlation lengths that are normal and parallel to the substrate surface, tilt and twist, and heterogeneous strain) by HXRD, and the interface structure of $\mathrm{AlN} / \mathrm{SiN}_{x} / \mathrm{Si}$ (111) and the dislocation densities (edge and screw types) by both TEM and XRD. The nitridation time varied from 0 to $660 \mathrm{~s}$.

\section{Experimental procedure}

GaN epitaxial layers on Si (111) substrate were grown in a low-pressure MOCVD reactor (Aixtron 200/4 HT-S). The reactant source materials for $\mathrm{Ga}, \mathrm{Al}$, and $\mathrm{N}$ were trimethylgallium (TMGa), trimethylaluminum (TMAl), and $\mathrm{NH}_{3}$, respectively. The $\mathrm{H}_{2}$ was used as a carrier gas during AlN and
$\mathrm{GaN}$ growth. Before loading, the $\mathrm{Si}$ substrates were sequentially degreased by $\mathrm{H}_{2} \mathrm{SO}_{4}: \mathrm{H}_{2} \mathrm{O}_{2}: \mathrm{H}_{2} \mathrm{O}(2: 1: 1)$ solutions for $1 \mathrm{~min}$, and etched while in a $2 \% \mathrm{HF}$ solution for $1 \mathrm{~min}$, rinsed in de-ionized water, and dried with a nitrogen gun. At the beginning of the growth of AlN, the substrate was baked in an $\mathrm{H}_{2}$ ambient at $1100^{\circ} \mathrm{C}$ for 10 min to remove the native oxide. To grow a $\mathrm{SiN}_{x}$ interlayer on a $\mathrm{Si}$ (111) substrate surface, following the thermal etching, the substrate was nitridated by exposing it to the $\mathrm{NH}_{3}$ flow of $0.900 \mathrm{slm}$ at $1020^{\circ} \mathrm{C}$. Nitridation was performed at five different times. The nitridation times were: 0 (without nitridation), 10, 60, 120, 420, and $660 \mathrm{~s}$ for samples A, B, C, D, E, and F, respectively. After the nitridation, for all the samples, we grew an approximately $150 \mathrm{~nm}$ high-temperature $\left(1100^{\circ} \mathrm{C}\right) \mathrm{AlN}(\mathrm{HT}$-AlN) buffer layer. In all of the samples, the $250 \mathrm{~nm}$ GaN layers were grown at $1050^{\circ} \mathrm{C}$. For sample A, in order to prevent the growth of an amorphous $\mathrm{SiN}_{x}$ interlayer, the technique of the $\mathrm{Al}$ pre-covering process of $\mathrm{Si}$ substrate was applied before the growth of the AlN buffer.

The crystalline quality of the GaN layers was examined by high resolution X-ray diffraction (HRXRD). The $\mathrm{X}$-ray diffraction was performed using a Bruker D-8 highresolution diffractometer system, delivering a $\mathrm{CuK} \alpha 1$ $(1.540 \AA)$ radiation. Data were collected on the (0002), (0004), (0006), (10 $\overline{1} 5),(20 \overline{2} 2),(12 \overline{3} 1),(10 \overline{1} 1),(10 \overline{1} 3)$, and $(11 \overline{2} 4)$ reflections.

HRTEM (High Resolution Transmission Electron Microscopy) was used to correlate the $\mathrm{X}$-ray measurements to the microstructure, crystal structure, and dislocations of $c$-plane $\mathrm{GaN}$ grown with $\mathrm{SiN}_{x}$ interlayer. TEM examinations were conducted on the plan-view and cross-sectional samples.

For the cross-sectional TEM investigations, all the samples were prepared via a conventional sandwich technique, i.e., mechanically thinning them to a thickness of approx. $\sim 5 \mu \mathrm{m}$ by dimple grinding. Gatan 691 Precision Ion Polishing System (PIPS) was used to prepare the thin foils.

The specimens were examined by using a JEOL 2100 High Resolution Transmission Electron Microscope $\left(\mathrm{LaB}_{6}\right.$ filament) operated at $200 \mathrm{kV}$.

\section{Results and discussion}

Figure 1 shows the typical $2 \theta$ scan X-ray diffraction (XRD) patterns of the GaN films grown on a nitridated (with $120 \mathrm{~s}$ nitridation time) $\mathrm{Si}$ (111) substrate. As shown in Fig. 1, the diffraction patterns only exhibited the dominant Wurtzite GaN crystalline (0002) peaks plus (0002) peaks from the AlN layer and (111) peaks from Si substrate. This indicates the hexagonal structure of the GaN epilayer with its [0001] direction parallel to the [111] of Si substrate. For sample D, the (0002) reflections of Wurtzite $\mathrm{GaN}$ are clearly observed at $17.162^{\circ}$ and the (111) peak from Si substrate at $17.161^{\circ}$, respectively. 


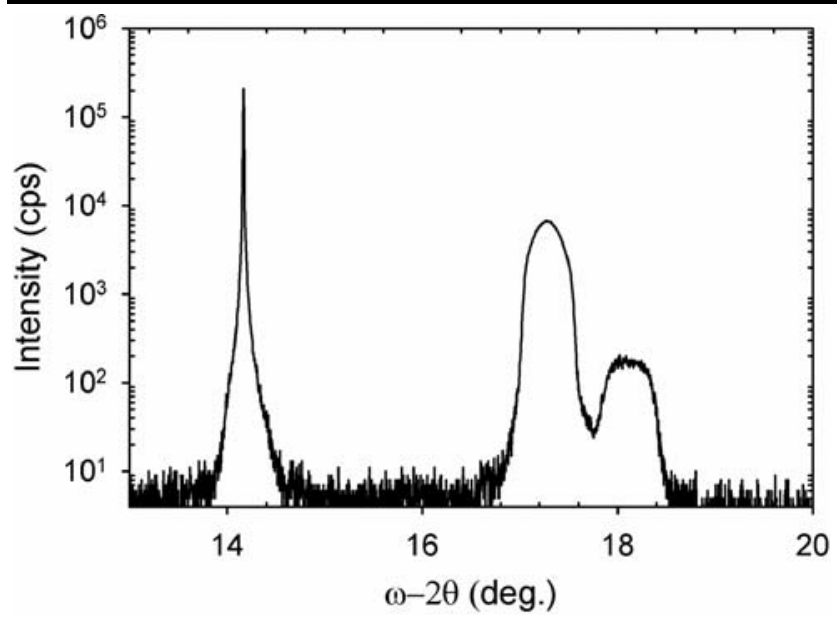

Fig. 1 An HR-XRD $\omega-2 \theta$ scan pattern obtained using four-Ge (022) crystal monochromotor from a GaN epilayer grown on Si (111) substrate with $120 \mathrm{~s}$ nitridation times (sample D)

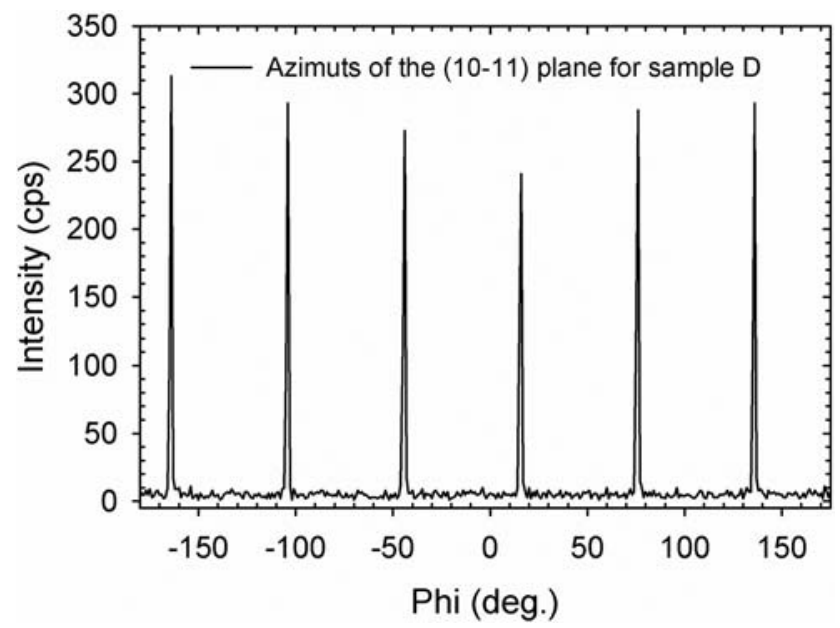

Fig. 2 Phi-scan curve of asymmetric GaN (1011) reflection plane for sample D. Every peak shows the azimuths of the (1011) plane. The diffractive peak repeats every $60^{\circ}$

An in-plane $\Phi$-scan was also taken by rotating the sample around its surface-normal direction in order to investigate the in-plane alignment of GaN film. Figure 2 shows the $\Phi$-scan pattern of the $(10 \overline{1} 1)$ plane of sample D. It can be seen in Fig. 2 that the diffraction peaks from the (10 $\overline{1} 1)$ plane of $\mathrm{GaN}$ were observed at $60^{\circ}$ intervals, which clearly confirms the good single crystal hexagonal structure of the $\mathrm{GaN}$ epilayer.

The influence of the nitridation time on the structural properties of a $\mathrm{GaN}$ epilayer

Heteroepitaxial thin films with a large lattice-mismatch with respect to the substrate in turn form a mosaic structure of slightly misoriented sub-grains [18, 19, 22], which is characterized by the nucleation of slightly misoriented islands and the coalescence of these islands towards a smooth surface. The mosaic structure of the epilayers is determined by the size and angular distribution of the mosaic blocks. The vertical and lateral correlation lengths, heterogeneous strain, and degree of mosaicity as expressed by the tilt and twist angles are important parameters in characterizing the quality of the epitaxial films with a large lattice mismatch to the substrate $[17,19,22]$. The mosaic blocks are assumed to be slightly misoriented with respect to each other. The outof-plane rotation of the blocks perpendicular to the surface normal is the mosaic tilt, and the in-plane rotation around the surface normal is the mosaic twist. The average absolute values of tilt and twist are directly related to the full width at half maximum (FWHM) of the corresponding distributions of the crystallographic orientations $[18,23]$.

The parameters of the vertical and lateral coherence length and tilt angle can be obtained from the WilliamsonHall measurement, and the twist angle from an approach, which was developed by Srikant et al. [23] or from direct measurement [24], which explains the superposed effect of the tilt and twist on the broadening of the full-widths at half-maximum (FWHMs) of the off-axis plane rocking curves [25].

Each contribution to the broadening of the particular XRD curves can be separated in the Williamson-Hall measurement $[18,25]$. Specifically, in triple-axis diffractometer measurements, the broadening of the rocking curve (angular-scan or $\omega$-scan) of the symmetric (0002), (0004), and (0006) reflections for the GaN epitaxial layer is influenced only by the tilt angle $\alpha_{\text {tilt }}$ and short coherence length parallel to the substrate surface $L_{\|}$.

Separation analogous of the tilt angle $\alpha_{\text {tilt }}$ and short coherence length can be made with the Williamson-Hall plot, when $\beta_{\Omega}(\sin \theta) / \lambda$ is plotted against $(\sin \theta) / \lambda$ for each reflection and fitted by a straight line. Then, the tilt angle $\alpha_{\text {tilt }}$ is obtained from the slope of the linear dependence, and lateral coherence length $L_{\|}\left(L_{\|}=0.9 /\left(2 y_{0}\right)\right)$ from the inverse of the $y$-intersection $y_{0}$ of the fitted line with the ordinate, where $\beta_{\Omega}$ is the FWHM in angular unit, $\theta$ is the Bragg reflection angle, and $\lambda$ is the $\mathrm{X}$-ray wavelength.

In the radial-scan direction $(\omega-2 \theta$-scan) of the symmetric reflections (0002), (0004), and (0006), a small vertical correlation length and a heterogeneous strain along the $c$-axis causes a broadening of the Bragg reflections. This two parameters $L_{\perp}$ and $\varepsilon_{\perp}$ can similarly be derived from the Williamson-Hall plot. The $L_{\perp}$ and $\varepsilon_{\perp}$ parameters can be derived from the $\beta_{2 \theta-\Omega}(\cos \theta) / \lambda$ against $(\sin \theta) / \lambda$ plots for each reflection.

Figure 3 shows the corresponding Williamson-Hall plots for reflections (0002), (0004), and (0006) for the triple-axis (a) $\omega$-scan and (b) $\omega-2 \theta$ of all the samples with different nitridation times. The straight lines are linear fits of the experimental data. The expected linear behavior of the graphs 

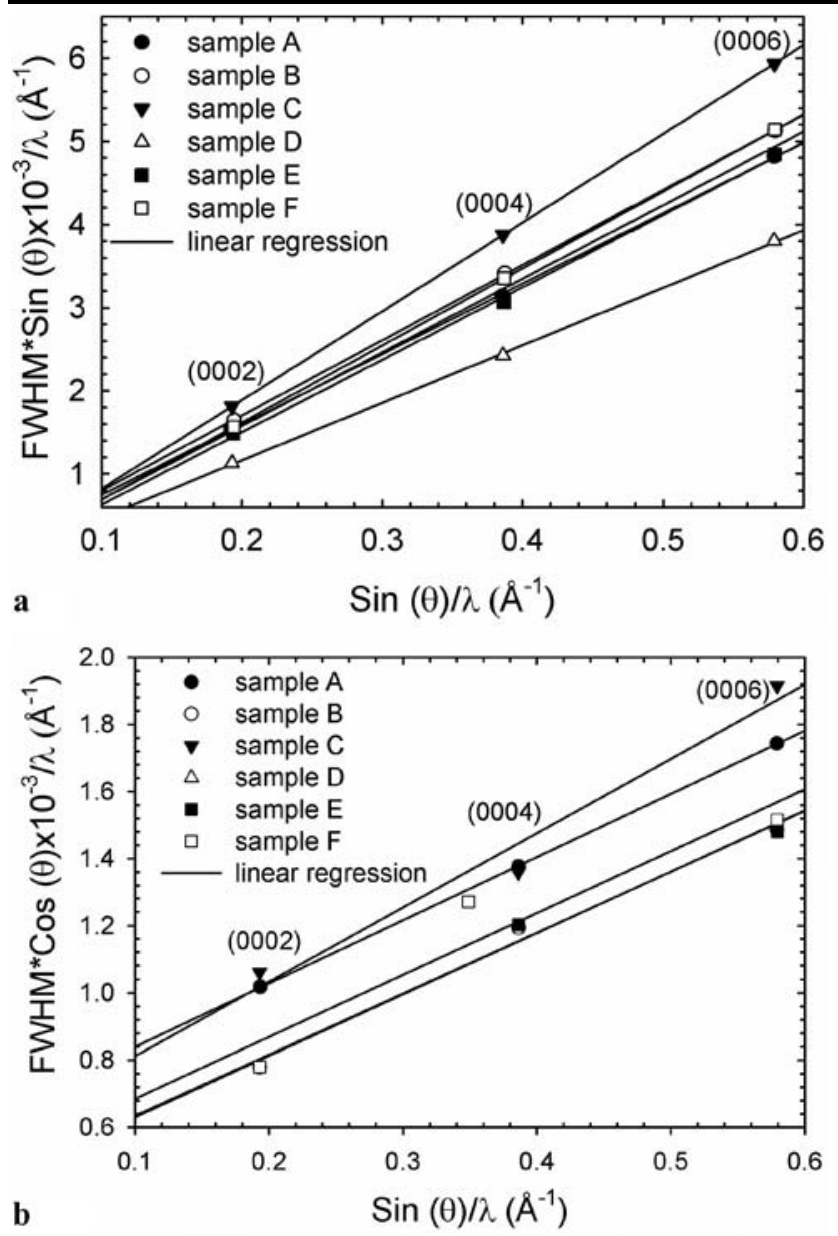

Fig. 3 Williamson-Hall plot for the GaN layers without nitridation and with different nitridation times. (a) Triple-axis $\omega$ scan and (b) triple-axis $\omega-2 \theta$ scan were measured for the $(000 l)(l=2,4,6)$ reflections indicated in the figure. The lines result from a linear fit of the data

is experimentally well confirmed, which gives rather accurate tilt angle values.

The lateral coherence lengths and tilt angles are shown in Fig. 4. As can be seen, the tilt angles for all the samples were rather small. It can be seen in the figure that, as the nitridation times increased, the tilt angles decreased first and then increased to $10.6 \times 10^{-3}$ degrees for sample $\mathrm{C}(60 \mathrm{~s})$. From these nitridation times, we can see small increments in the tilt angle. The minimum tilt angle was obtained for sample D. The lateral coherence lengths were determined to range from 182 to $467 \mathrm{~nm}$. As seen in Fig. 4, as the nitridation times increased the $L_{\|}$increased first and then decreased. The maximum values were observed for the nitridation times of $10 \mathrm{~s}$ (sample B).

The vertical coherence lengths $L_{\|}$values are shown in Fig. 5. Its values range from 60 to $100 \mathrm{~nm}$. It can be seen in Fig. 5 that there is an increment in all the samples except for sample D.

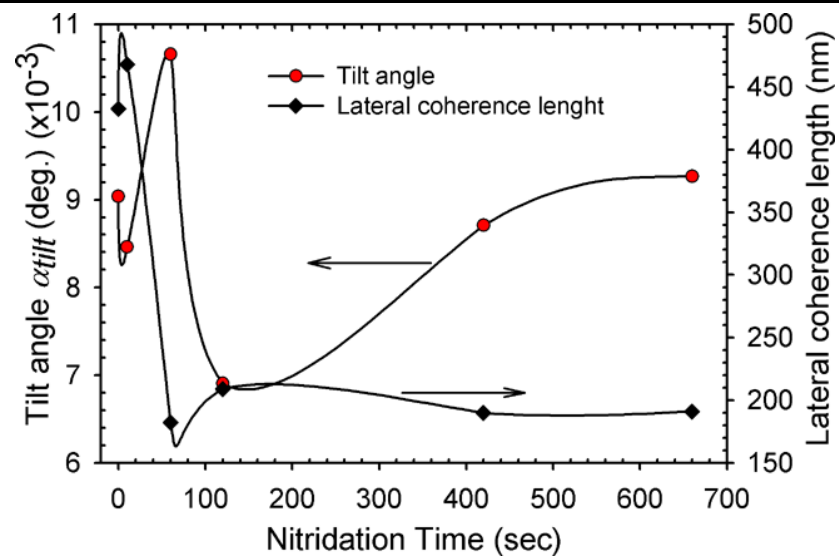

Fig. 4 Tilt and lateral coherence length obtained from the Williamson-Hall plot as a function of the nitridation time

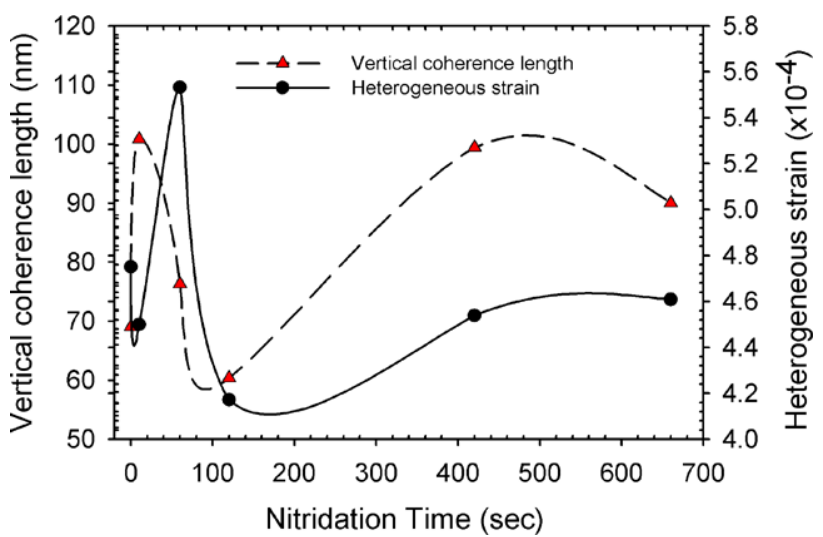

Fig. 5 Vertical coherence length and heterogeneous strain obtained from the Williamson-Hall plot as a function of the nitridation time

The strain normal to the substrate $\varepsilon_{\perp}$ values, which was obtained for all the samples, is shown in Fig. 5. The $\varepsilon_{\perp}$ values range from $4.2 \times 10^{-4}$ to $5.5 \times 10^{-4}$. Small changes were observed for a heterogeneous strain with the nitridation time length.

The mean twist angle between the subgrains can be extrapolated from a fit to the measured double-axis scans data for different (hkl) reflections in the skew symmetric diffraction. Several extrapolation methods have been reported in the literature for the mean twist angle calculation [18, 23, 26]. However, all of these methods include a complicated calculation and fitting procedure for the extraction of the twist angle from the experimental data. On the other hand, Zheng et al. [24] proposed a simple empirical approach to obtain the mean twist angle directly without falling into complications.

The FWHM of the rocking curve of an imperfect film is composed of several contributions, such as the mean tilt, twist, the average size of sub-grains, and inhomogeneous strain distributions. Although the broadening, which is due to a limited domain size and an inhomogeneous strain, can 


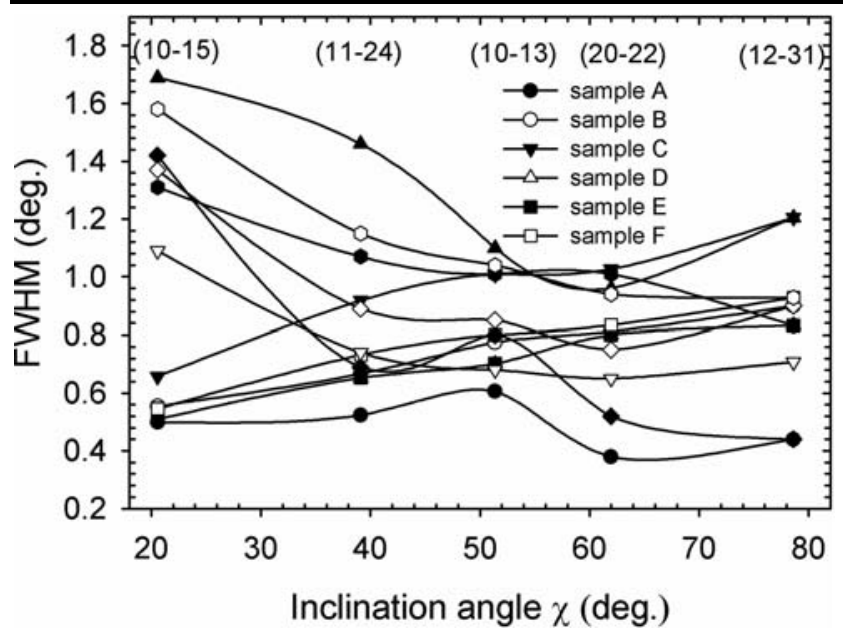

Fig. 6 FWHM values of the rocking curves of $\Phi$ and $\omega$ scans for (hkl) reflections (marked in the figure) vary with the inclination angle $\chi$ for all of the samples. FWHMs of $\Phi$ scan increase with the increment of $\chi$ angle, while those of $\omega$ scan decrease. The lines are a guide for the eyes

be significant in highly imperfect films, their effects have been eliminated by the use of a slit of $0.6 \mathrm{~mm}$ placed in front of the detector in double-axis $\omega$-scans. Indeed, their contribution to the overall broadening was found to be of minor influence in this measurement case.

In addition, the (0002) reflection and (hkl) reflections with $\mathrm{h}$ or $\mathrm{k}$ non-zero orientation of our samples with tripleaxis $\omega-2 \theta$-scans both exhibit a small FWHM. The last important point is that the intrinsic width of the reflection for the crystal and the apparatus broadening for all the experimental reflections are negligible because these effects amount to only a few arcsec. For this reason, we can only measure the broadening that is caused by the twist by using (hkl) reflections in the skew geometry.

The extended FWHMs of $\omega$ and $\Phi$-scans that were obtained by using the fit of the Pseudo-Voigt function to the rocking curves are shown in Fig. 6 as a function of increasing $\chi$ for all the samples. It can be seen in Fig. 6 that the FWHMs of $\omega$ and $\Phi$-scans are nearly the same when the inclination angle is $78.6^{\circ}$. In this inclination angle, the $(12 \overline{3} 1)$ reflection appears. These results showed that the rockingcurve widths of $\omega$ or $\Phi$ scans for this higher $\chi$ angle are close to the twist angles. In every respect, the FWHMs of $\Phi$-scans are larger than those of the $\chi$-scans with the change of the inclination angle $\chi$. Therefore, the mean twist angles must be the average value of the FWHMs of the $\omega$ and $\Phi$-scans of $\chi=78.6^{\circ}$.

The measured mean twist angle of the GaN layers is shown in Fig. 7 as a function of the nitridation time. The mean twist angle is strongly dependent on the nitridation time. The minimum mean twist angle value was obtained as $0.4^{\circ}$ for sample A (without nitridation). It increases with the nitridation time and reaches to a maximum value of $1.2^{\circ}$ for

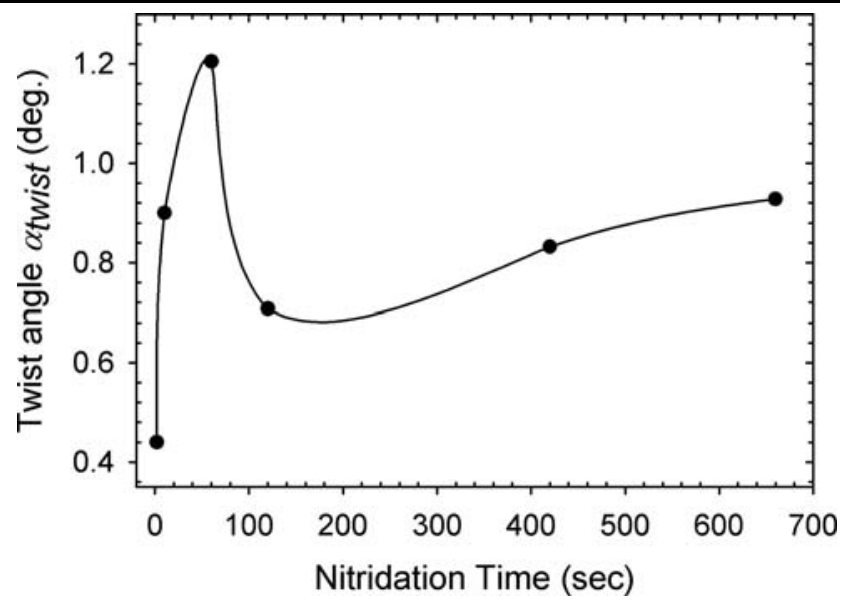

Fig. 7 The dependence of the twist angle on the nitridation time

sample $\mathrm{C}$ (with $60 \mathrm{~s}$ nitridation) and then decreases to $0.7^{\circ}$ for sample D (with $120 \mathrm{~s}$ nitridation) nitridated the longest. Based on this observation, it can be argued that the mean twist angle of the GaN epilayers that are grown on Si substrate is strongly affected by the time elapsed for the nitridation of the substrate before intiating GaN growth.

There are three main types of dislocations present in GaN thin film that are grown on $\mathrm{Si}$ (111) with two steps [7-16, 18, 24, 27]: the pure edge dislocation with Burgers vector $b=\frac{1}{3}\langle 11 \overline{2} 0\rangle(\langle a\rangle)$, the pure screw dislocation with Burgers vector $b=\langle 0001\rangle(\langle c\rangle)$, and the mixed dislocation with $b=\frac{1}{3}\langle 11 \overline{2} 3\rangle(\langle c+a\rangle)$. The coherence lengths, and the twist and tilt are correlated with the different types of dislocations. As reported by Metzger et al. [18], for (0002)-oriented GaN epitaxial films, the mean twist angle is monotonically related to the density of edge-type of the threading dislocation (TD) with Burgers vector $b=$ $1 / 3\langle 11 \overline{2} 0\rangle$, while the mean tilt angle is monotonically related to, and can be converted into, the density of the screwtype of TD with Burgers vector $b=\langle 0001\rangle$. Thus, in the present study, all the existing dislocation lines were treated as the types defined by Metzger et al. [18].

The screw dislocation $(b=\langle 0001\rangle)$ density ( $\left.D_{\text {screw }}\right)$ can be calculated from the equation $[28,29]$,

$D_{\text {screw }}=\frac{\alpha_{\text {tilt }}^{2}}{4.35\left|b_{\text {screw }}\right|^{2}}$,

where $\alpha_{\text {tilt }}$ is the tilt angle, and $b$ is the Burgers vector length $\left(b_{\text {screw }}=0.5185 \mathrm{~nm}\right)$.

The edge type dislocations with Burgers vector $(b=$ $\left.\frac{1}{3}\langle 11 \overline{2} 0\rangle\right)$ reconcile an azimuthal rotation of crystallites around the surface normal. From the measured twist angle $\alpha_{\text {twist }}$, an edge-type dislocation density can be calculated. If dislocations are piled up in small angle grain boundaries, 


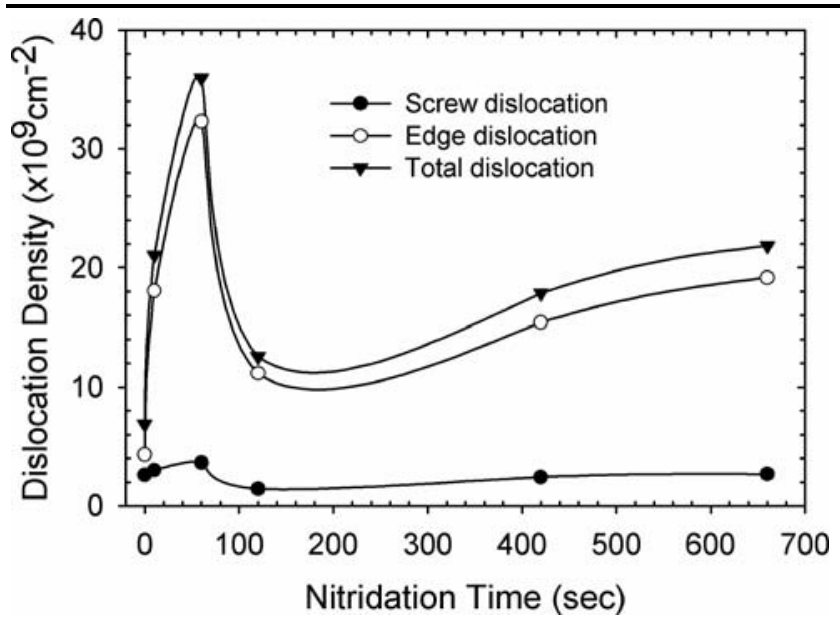

Fig. 8 The edge, screw, and total dislocation density as a function of the nitridation time

the edge dislocation density ( $D_{\text {edge }}$ ), therefore, can be calculated according to $[28,29]$,

$D_{\text {edge }}=\frac{\alpha_{\phi}}{2.1\left|b_{\text {edge }}\right| L_{\|}}$,

where $b$ is the Burgers vector length $\left(b_{\text {edge }}=0.3189 \mathrm{~nm}\right)$. Figure 8 shows the edge, screw, and total dislocation density as a function of nitridation time length. As can be seen from this figure, the edge and screw dislocation densities are approximately equal to each other for sample A (without nitridation). The edge dislocation density changed with the nitridation times. On the other hand, the screw dislocation densities approximately keep constant for all the nitridation times. These results show that the nitridation process is much more effective on the edge type dislocation density.

The microstructure of the grown layers was revealed by TEM. The cross-sectional and plan-view TEM specimens were prepared from the grown layers. In Fig. 9(a), we show the cross-sectional TEM images of the GaN films grown on $\mathrm{Si}$ (111) with a $660 \mathrm{~s}$ nitridated buffer layer (sample F). The

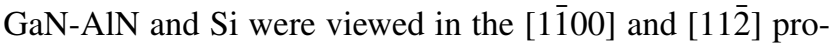
jections, respectively. A relatively rough interface between the HT-AIN nucleation layer and GaN, and a high density of threading dislocations (TD) can be clearly observed. From Fig. 9(a), it can be seen that, the dislocation density decreases rapidly within $50 \mathrm{~nm}$ from the AlN/GaN interface into the GaN layer. Since dislocations cannot end within a crystal, that is, the ends of a dislocation can only be at free surfaces, or at interfaces, the apparent change in the dislocation density within the GaN layer requires an explanation. The dislocations originating from the GaN/AlN interface may change direction and bend back to the interface that they originate from. Tilt or twist boundaries that are said to exist within the GaN layer may also assist in such changes in dislocation directions. Some studies even suggest the presence of small angle grain boundaries with the
GaN layer [18]. The wavy nature of the AlN/GaN interface may also be related to the tilt and twist boundaries in the GaN layer.

Other aspects requiring explanation are the crystallographic relationship between the AlN layer and Si substrate, as well as the apparent stress that consequently also leads to the observed high dislocation density within the AlN layer. If the interface was truly between the AlN and Si substrate, the crystallographic relationship and relatively high dislocation density, originating from the interface due to the large lattice mismatch between the two crystals, would provide a straightforward explanation. However, the AlN/Si interface has been shown, in the present study and others, to contain an amorphous $\operatorname{SiN}_{x}$ layer [34, 36, 37]. The orientation relationships were determined for GaN-AlN on $\mathrm{Si}$ (111) by taking the selected area electron diffraction (SAED) patterns in TEM (Fig. 9(b) and Fig. 10(b), (c)). It is noteworthy that despite the presence of an amorphous $\mathrm{SiN}_{x}$ interlayer, the selected area electron diffraction (SAED) confirms the well-known orientation relationships between $\mathrm{GaN}$ (AlN) and Si [34], which are $\langle 11 \overline{2} 0\rangle_{\mathrm{GaN}} / /\langle 11 \overline{2} 0\rangle_{\mathrm{AlN}} / /$ $\langle 1 \overline{1} 0\rangle_{\mathrm{Si}},\langle 1 \overline{1} 00\rangle_{\mathrm{GaN}} / /\langle 1 \overline{1} 00\rangle_{\mathrm{AlN}} / /\langle 11 \overline{2}\rangle_{\mathrm{Si}}$, and $\langle 0001\rangle_{\mathrm{GaN}}$ $/ /\langle 0001\rangle_{\mathrm{AlN}} / /\langle 111\rangle_{\mathrm{Si}}$. By this, it has also been proven that $\mathrm{GaN}$ and AlN have hexagonal crystal structures and are single crystals.

Several reports have addressed the issue of a crystallographic relationship between AIN and Si despite the amorphous $\operatorname{SiN}_{x}$ between them. One of the suggestions was that the amorphous $\mathrm{SiN}_{x}$ layer has some short range order that necessitates the orientation of the AlN layer in this fashion [34]. According to some others, there exists in the $\operatorname{SiN}_{x}$ a top layer of a few atomic distances that is crystalline [36]. According to Dobos et al. [37], Nakada et al. [36], and Kaiser et al. [34], the epitaxial relationship is transferred from $\mathrm{Si}$ into $\mathrm{AlN}$ and $\mathrm{GaN}$ through the amorphous $\mathrm{SiN}_{x}$ layer by the presence of crystalline regions, $\mathrm{Si}_{3} \mathrm{~N}_{4}$ inclusions, or holes in the $\mathrm{SiN}_{x}$ layer. Other explanations include the short-range tetrahedral coordination that was maintained in the $\mathrm{SiN}_{x}$ layer [34, 41, 42], and the nucleation and growth of $\mathrm{GaN}$ islands from intrinsic or extrinsic defects by a "pinhole model" [40].

In our view, perhaps the most plausible explanation is that the amorphous $\mathrm{SiN}_{x}$ layer is not continuous and that there must be some points of actual contact between the crystalline Si substrate and deposited AlN layer [40]. It is then conceivable to assume that such locations of contact would inevitably behave like seeds in bulk single crystal growth techniques in turn leading to an overall crystallographic relationship between AlN and Si despite the presence of an intermittent amorphous $\mathrm{SiN}_{x}$. If such a mode of deposition is operational, then the large lattice mismatch between AlN and $\mathrm{Si}$ in the locations of true contact could also act as a source for dislocation emissions, and thereby explain the 
Fig. 9 (a) Cross-sectional

Bright Field TEM image, and

(b) corresponding diffraction pattern for GaN-AlN [1] 100$]$

zone axis and $\mathrm{Si}$ [112] zone axis
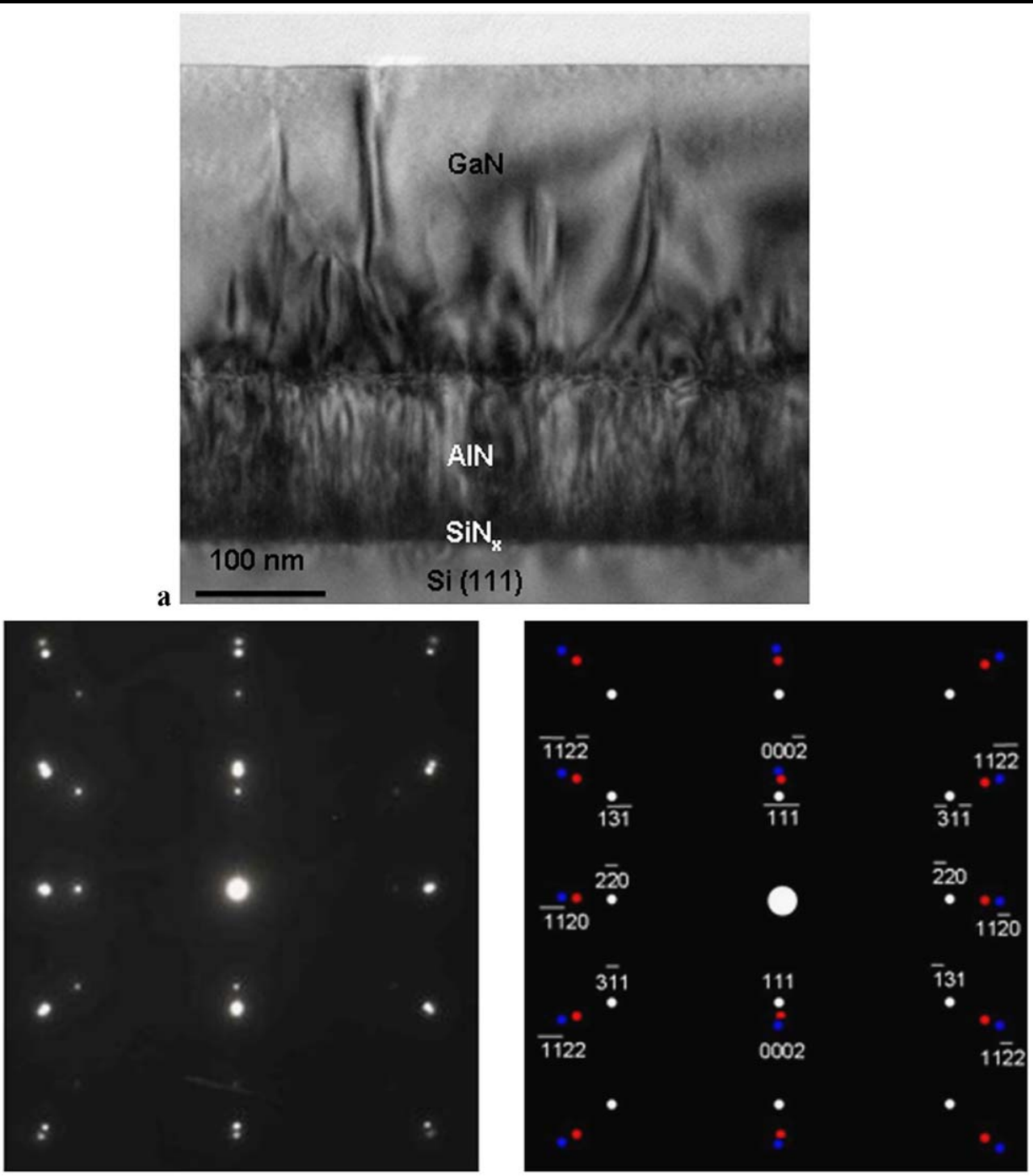

b

\section{- Si}

AIN high dislocation density within AlN. Our observations on the cross sections taken from different locations of the same sample showed that $\mathrm{SiN}_{x}$ is indeed intermittent. It may further be suggested that the tilt and twist boundaries that are said to exist in GaN may also be related to the growth modes of the AlN layer.

In addition to the arguments mentioned above, the crystal orientation of the hexagonal AIN with the basal planes parallel to its interface with the substrate may also arise from the simple thermodynamic requirement of lowering the surface energy. As a thin film and, therefore, having its largest surfaces parallel to the substrate it should be expected that AlN with a hexagonal crystal structure would, unless otherwise dictated by a crystalline substrate, prefer to form outer surfaces out of densely packed, i.e., low energy, basal planes. Therefore, even if no effect was imposed by the amorphous $\mathrm{SiN}_{x}$ for a particular orientation relationship, a free-growing
AlN layer would be expected to position itself in the observed fashion, i.e., basal planes parallel to the substrate.

A plain AlN film on Si contains a large number of dislocations due to the strain relaxation of the film [30]. Follstaedt et al. [31] and Datta et al. [32] have demonstrated that, plan-view TEM imaging is generally accepted as a more trustworthy method for TD density measurement, compared to other techniques, such as atomic force microscopy (AFM) and cathodoluminescence (CL). These techniques cannot be used effectively to detect a-type edge dislocations that thread to the film surface, since such dislocations do not create steps on the surface that are detectable by AFM, and are probably not effective nonradiative recombination centers that are detectable by CL [31-34].

The threading dislocations (TD) density of sample $\mathrm{F}$ (with $660 \mathrm{~s}$ nitridation) was determined by plan-view TEM. The plan-view image of sample F is shown in Fig. 10(a). All 


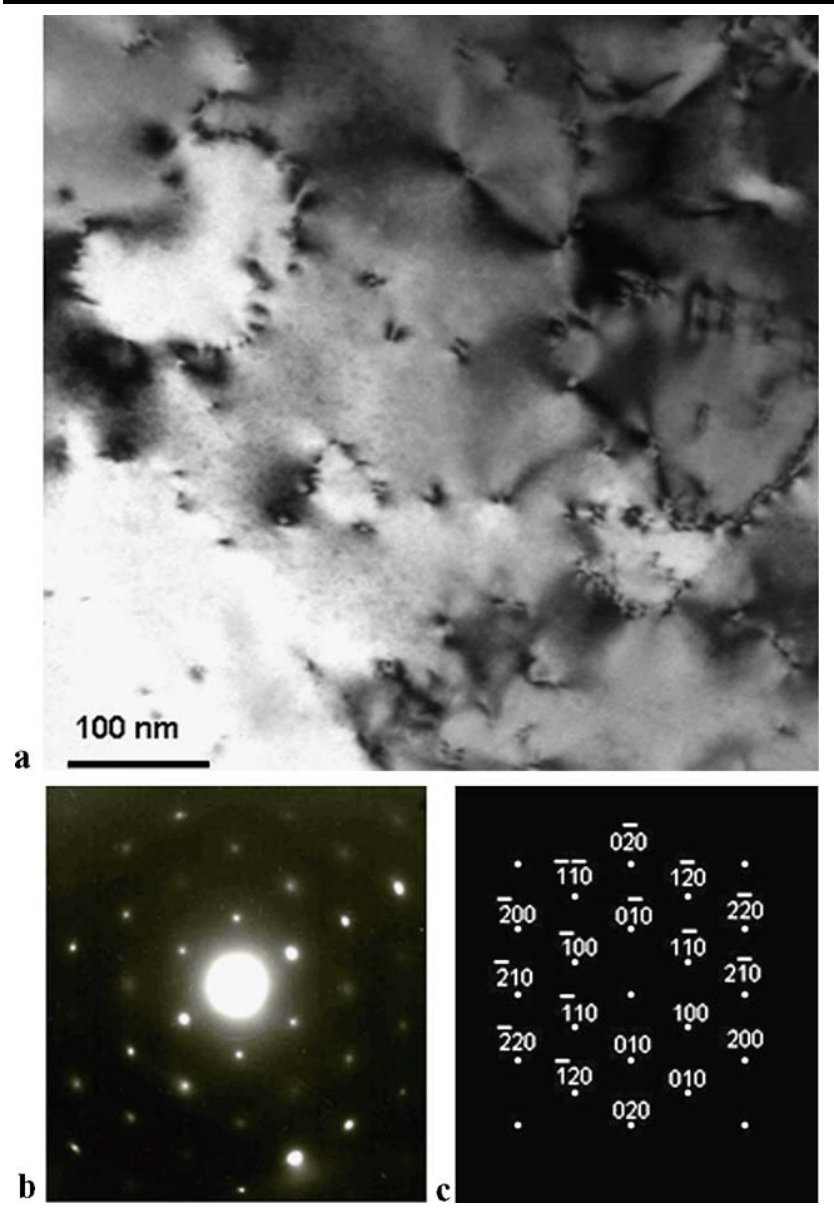

Fig. 10 (a) Plan-view TEM micrographs of the GaN layer on the AIN nucleation overlayer grown with the insertion of an $\mathrm{SiN}_{x}$ layer (sample F) and (b) corresponding diffraction pattern, and (c) indexed sketch of the basic reflections for the GaN [0001] zone axis

of the images were bright-field (BF) images which were collected by using multi-beam diffraction conditions. The planview specimens were investigated in the [0001] zone-axis orientation, which were nearly in the optical axis. The determined values are characteristic for the near surface region, only ( top $100 \mathrm{~nm}$ ). Moreover, the crystal defects, which were revealed by cross-sectional studies, did not propagate to the surface, and are not visible in the plan-view images [30, 34, 35].

As shown in Fig. 10(a), a network structure of defect arrays was also observed. The image shows that the dislocations in the epitaxial layers are not uniformly distributed. In order to determine the TD densities, the number of TDs was counted for approximately 10 different plan-view TEM images similar to those shown in Fig. 10. All of the images, for TD density determination, were collected with their GaN [0001] zone axis. The density of these threading dislocations was approximately $9 \times 10^{9} \mathrm{~cm}^{-2}$, which is comparable to the values that were calculated with the HR-XRD measurements.
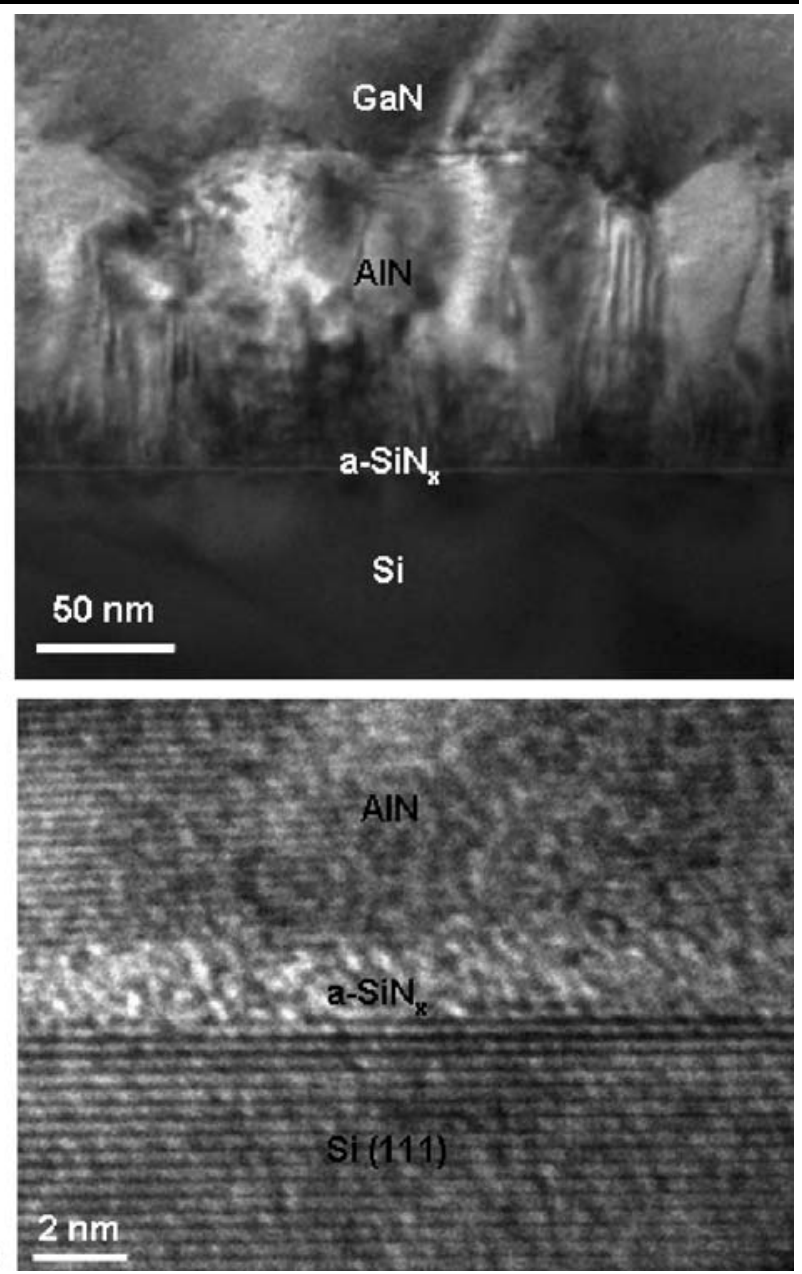

Fig. 11 (a) Low-magnification and (b) HRTEM image of the Si/AIN interface with the $\mathrm{a}-\mathrm{SiN}_{x}$ intermediate layer

Between the Si substrate and AlN layer, a thin layer ( $\sim 2 \mathrm{~nm}$ ) was observed (Fig. 11). From the cross-sectional High Resolution TEM (HRTEM) image (Fig. 11(b)) for sample $\mathrm{F}$ it is observed that this thin layer is amorphous $\mathrm{SiN}_{x}\left(\mathrm{a}-\mathrm{SiN}_{x}\right)$. This a-SiN $x$ is also observed in several Si/AlN systems [33, 34, 36-42].

Cross-sectional HRTEM images are shown for the GaN/AIN interface in Fig. 12. A rough interface is observed mostly for the GaN/AlN layers (Fig. 11(a) and Fig. 12(a)). However, smoother interface segments were also observed in some regions (Fig. 12(b), (c)).

From the SAED results, with the epitaxial relation, it can be observed that the crystal orientation of $\mathrm{Si}$ (111) seems to be transferred mostly through the amorphous $\mathrm{SiN}_{x}$ into the AlN and GaN layers. One reason could be the short-range tetrahedral coordination of each atom, which is obviously continued in the $\mathrm{SiN}_{x}$ layer. Another explanation could be small crystalline $\mathrm{SiN}$ or $\mathrm{Si}_{3} \mathrm{~N}_{4}$ within the interlayer, which transfer the orientation relation $[34,36]$. Secondly, a locally, thicker $\mathrm{SiN}_{x}$ layer in the structures can cause the interrup- 

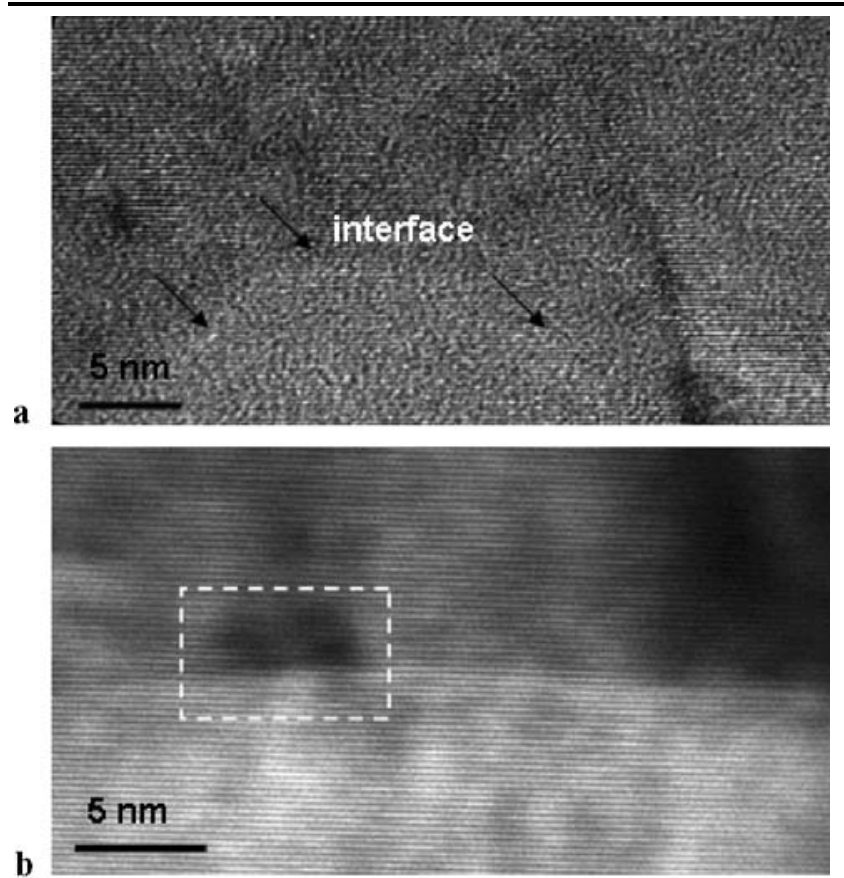

b

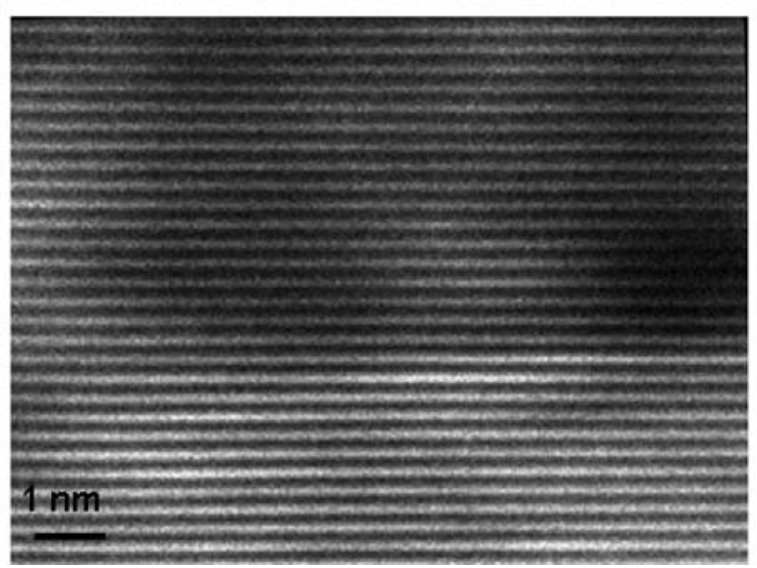

Fig. 12 (a) HRTEM image of the rough AlN/GaN interface shown by the arrows, (b) HRTEM image of the smoother AIN/GaN interface region, and (c) magnified view of the selected region of $(\mathbf{b})$

tion of the crystal symmetry transfer. For this reason, we obtained (0002) orientated AlN grains (Fig. 9) with slightly tilted grains. In our present study, it was important to avoid an excessively large $\mathrm{SiN}_{x}$ layer thickness that would totally interrupt the transfer of the epilayer relation from the $\mathrm{Si}$ (111) substrate to the epitaxial layer and that would cause a polycrystalline deposition of $\mathrm{GaN}$, as given by other growth process [13-16, 31, 33-35].

\section{Conclusions}

In the present study, the influence of in-situ substrate nitridation time (assumed to cause an $\operatorname{SiN}_{x}$ amorphous interlayer) on the characteristics of the structural features by HXRD, and the interface structure of $\mathrm{AlN} / \mathrm{SiN}_{x} / \mathrm{Si}$ (111) and dislocation densities (edge and screw types) were investigated by both TEM and XRD measurements of the hexagonal epitaxial GaN films grown on $\mathrm{Si}$ (111). The nitridation time lengths ranged from 0 to $660 \mathrm{~s}$. The mosaic structure of the GaN layers was affected by the nitridation time length. We obtained the largest values for vertical and lateral coherence length at $10 \mathrm{~s}$ nitridation. On the other hand, when the $\mathrm{Si}$ (111) was not nitridated, the smallest twist angle was obtained (sample A). The smallest tilt angle of a $6.9 \times 10^{-3}$ degree was obtained for the $120 \mathrm{~s}$ nitridated sample. From the dislocation measurements, we can see that the edge type dislocation densities are much more affected from the nitridation process. However, screw dislocation densities are less affected by nitridation. From the SAED results, with the epitaxial relation, it can be observed that the crystal orientation of $\mathrm{Si} \mathrm{(111)} \mathrm{seems} \mathrm{to} \mathrm{be} \mathrm{transferred} \mathrm{mostly} \mathrm{through} \mathrm{the} \mathrm{amor-}$ phous $\mathrm{SiN}_{x}$ into the AlN and GaN layers. However, it is important to avoid an excessively large $\mathrm{SiN}_{x}$ layer thickness, which would totally interrupt the transfer of the epilayer relation from the $\mathrm{Si}$ (111) substrate to the epitaxial and would cause the polycrystalline deposition of $\mathrm{GaN}$.

Acknowledgement This work is supported by the European Union under the projects EU-METAMORPHOSE, EU-PHOREMOST, EUPHOME, and EU-ECONAM, and TUBITAK under Project Numbers 105E066, 105A005, 106E198, 106A017, and 107A012. One of the authors (E.O.) also acknowledges partial support from the Turkish Academy of Sciences.

\section{References}

1. S.N. Mohammad, A. Salvador, H. Morkoç, Proc. IEEE 83, 1420 (1996)

2. L. Shen, S. Heikman, B. Moran, R. Coffie, N.-Q. Zhang, D. Buttari, I.P. Smorchkova, S. Keller, S.P. DenBaars, U.K. Mishra, IEEE Electron Device Lett. 22, 457 (2001)

3. S. Butun, M. Gokkavas, H. Yu, E. Ozbay, Appl. Phys. Lett. 89, 073503 (2006)

4. T. Tut, M. Gokkavas, B. Butun, S. Butun, E. Ulker, E. Ozbay, Appl. Phys. Lett. 89, 183524 (2006)

5. H. Yu, D. Caliskan, E. Ozbay, J. Appl. Phys. 100, 033501 (2006)

6. R. Gaska, J.W. Yang, A. Osinsky, Q. Chen, M. Asif Khan, A.O. Orlov, G.L. Snider, M.S. Shur, Appl. Phys. Lett. 72, 707 (1998)

7. A. Dadgar, C. Hums, A. Diez, J. Bläsing, A. Krost, J. Cryst. Growth 297, 279 (2006)

8. S. Pal, C. Jacob, Bull. Mater. Sci. 27, 501 (2004)

9. A. Krost, A. Dadgar, Mater. Sci. Eng. B 93, 77 (2002)

10. M.-H. Kim, Y.-G. Do, H.C. Kang, D.Y. Noh, S.-J. Park, Appl. Phys. Lett. 79, 2713 (2001)

11. Z.L. Fang, S.P. Li, J.C. Li, H.Z. Sun, S.J. Wang, J.Y. Kang, Thin Solid Films 516, 6344 (2008)

12. K. Cheng, M. Leys, S. Degroote, B. Van Daele, S. Boeykens, J. Derluyn, M. Germain, G. Van Tendeloo, J. Engelen, G. Borghs, J. Electron. Mater. 35, 592 (2006)

13. S. Raghavan, J.M. Redwing, J. Appl. Phys. 98, 023514 (2005)

14. J. Huang, Z. Ye, L. Wang, J. Yuan, B. Zhao, H. Lu, Solid-State Electron. 46, 1231 (2002) 
15. W.-Y. Uena, Z.-Y. Li, S.-M. Lan, S.-M. Liao, J. Cryst. Growth 280 , $335(2005)$

16. C.-L. Wu, J.-C. Wang, M.-H. Chan, T.T. Chen, S. Gwo, Appl. Phys. Lett. 83, 4530 (2003)

17. P.R. Hageman, S. Haffouz, V. Kirilyuk, A. Grzegorczyk, P.K. Larsen, Phys. Stat. Sol. (a) 188, 523 (2001)

18. T. Metzger, R. Höppler, E. Born, O. Ambacher, M. Stutzmann, R. Stömmer, M. Schuster, H. Göbel, S. Christiansen, M. Albrecht, H.P. Strunk, Philos. Mag. A 77, 1013 (1998)

19. B. Heying, X.H. Wu, S. Keller, Y. Li, D. Kapolnek, B.P. Keller, S.P. DenBaars, J.S. Speck, Appl. Phys. Lett. 68, 643 (1995)

20. M.E. Vickers, M.J. Kappers, R. Datta, C. McAleese, T.M. Smeeton, F.D.G. Rayment, C.J. Humphreys, J. Phys. D Appl. Phys. 38, 99 (2005)

21. N.G. Weimann, L.F. Eastman, J. Appl. Phys. 83, 3656 (1998)

22. V. Holy, J. Kubena, E. Abramof, K. Lischka, A. Pesek, E. Koppensteiner, J. Appl. Phys. 74, 1736 (1993)

23. V. Srikant, J.S. Speck, D.R. Clarke, J. Appl. Phys. 82, 4286 (1997)

24. X.H. Zheng, H. Chen, Z.B. Yan, Y.J. Han, H.B. Yu, D.S. Li, Q. Huang, J.M. Zhou, J. Cryst. Growth 255, 63-67 (2003)

25. G.K. Williamson, W.H. Hall, Acta Metall. 1, 22 (1953)

26. Y.J. Sun, O. Brandt, K.H. Ploog, J. Mater. Res. 18, 1247 (2003)

27. H. Sasaki, S. Kato, T. Matsuda, Y. Sato, M. Iwami, S. Yoshida, J. Cryst. Growth 298, 305 (2007)

28. P. Gay, P.B. Hirsch, A. Kelly, Acta Metall. 1, 315 (1953)

29. C.G. Dunn, E.F. Koch, Acta Metall. 5, 548 (1957)

30. H. Lahreche, P. Vennegues, B. Beaumont, P. Gibart, J. Cryst. Growth 205, 245 (1999)
31. D.M. Follstaedt, P.P. Provencio, N.A. Missert, C.C. Mitchell, D.D. Koleske, A.A. Allerman, C.I.H. Ashby, Appl. Phys. Lett. 81, 2758 (2002)

32. R. Datta, M.J. Kappers, J.S. Barnard, C.J. Humphreys, Appl. Phys. Lett. 85, 3411 (2004)

33. X. Weng, S. Raghavan, J.D. Acord, A. Jain, E.C. Dickey, J.M. Redwing, J. Cryst. Growth 300, 217 (2007)

34. S. Kaiser, M. Jakob, J. Zweck, W. Gebhardt, O. Ambacher, R. Dimitrov, A.T. Schremer, J.A. Smart, J.R. Shealy, J. Vac. Sci. Technol. B 18(2), 733 (2000)

35. F. Wu, S. Zamir, B. Meyler, J. Salzman, Y. Golan, J. Electron. Mater. 31, 88 (2002)

36. Y. Nakada, I. Aksenov, H. Okumura, Appl Phys Lett. 73, 6951 (1998)

37. L. Dobos, B. Pécz, E. Feltin, B. Beaumont, P. Gibart, Vacuum 71, 285 (2003)

38. M.-H. Kim, Y.-C. Bang, N.-M. Park, C.-J. Choi, T.-Y. Seong, S.-J. Park, Appl. Phys. Lett. 78, 2858 (2001)

39. A. Chakraborty, K.C. Kim, F. Wu, J.S. Speck, S.P. DenBaars, U.K. Mishra, Appl. Phys. Lett. 89, 041903 (2006)

40. M. Tamura, M. López-López, T. Yodo, Superficies Vacío 13, 80 (2001)

41. Z. Fang, Surf. Coat. Technol. 202, 4198 (2008)

42. Z.L. Fang, J.Y. Kang, W.J. Huang, H.T. Sun, M. Lu, J.F. Kong, W.Z. Shen, J. Phys. Chem. C 112, 4925 (2008) 